\title{
Procedure of Direct Hepatic Artery Puncture for the Treatment of Hepatocellular Carcinoma: Two Case Reports
}

\author{
Hidetaka Takashima ${ }^{a}$ Michihisa Moriguchi ${ }^{b}$ Kohichiroh Yasui ${ }^{b}$ Natsuko Hayashic \\ Kyohei Ikeda ${ }^{a}$ Kiyoshi Ogiso ${ }^{a}$ Chihiro Yokomizo ${ }^{a}$ Hirokazu Uejima ${ }^{a}$ \\ Hideo Tomioka ${ }^{a}$ Tadashi Itoh $^{a}$ Shigeto Mizuno ${ }^{d}$ Seiji Shimizu ${ }^{a}$ Yoshito Itoh $^{b}$ \\ aDepartments of Gastroenterology and Hepatology, Osaka General Hospital of West Japan \\ Railway Company, Osaka, Japan; bepartment of Molecular Gastroenterology and Hepatology, \\ Kyoto Prefectural University of Medicine Graduate School of Medical Science, Kyoto, Japan; \\ 'Department of Radiology, Osaka General Hospital of West Japan Railway Company, Osaka,

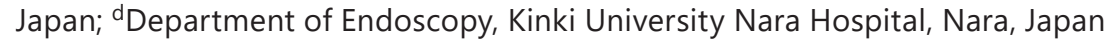

\author{
Keywords \\ Hepatocellular carcinoma - Direct hepatic artery puncture - Lipiodol · Ethanol injection . \\ Tumor rupture
}

\begin{abstract}
Recently, treatment options for hepatocellular carcinoma (HCC) have expanded due to the development of the tyrosine kinase inhibitor ramucirumab and immune checkpoint inhibitors. Transcatheter arterial chemoembolization is the standard therapy for intermediate-stage HCC; however, in cases with anatomical problems, normal approaches are not possible. In such rare cases, direct hepatic puncture may be considered as an effective therapy and an indispensable treatment. We report our novel method of direct hepatic artery puncture in this case report. In 2011 and 2017, we reported 2 cases in the journal of the Japan Society of Hepatology in Japanese. This therapy is difficult and is associated with a high risk of complications; however, we succeeded in both cases in a similar way. We believe this method may provide an alternative treatment when standard treatment is not possible or when urgent therapy is required. In case 1 , direct hepatic artery puncture was performed under ultrasonographic guidance, and we were able to control the disease with percutaneous lipiodol chemotherapy. Case 2 was an emergency case of ruptured HCC. Direct hepatic puncture successfully stopped tumor bleeding; furthermore, tumor necrosis also occurred, as seen on the enhanced computed tomography image. Our new method requires advanced puncture techniques and is not the treatment of choice if there are other safe alternatives available. However, it can be considered as an option if there are no other viable, effective treatments.
\end{abstract}




\section{Introduction}

Transcatheter arterial chemoembolization (TACE) is commonly used in hepatocellular carcinoma (HCC) unsuitable for resection, ablation, or liver transplantation as a bridge therapy [1]. The aim of TACE is to kill tumor cells with hypoxic damage and chemotherapeutic drugs (doxorubicin, epirubicin, cisplatin, or mitomycin C), using a carrier agent. Systemic therapy for HCC changed drastically after the introduction of the molecular targeted agent sorafenib in 2007. Sorafenib is a multi-tyrosine kinase inhibitor (TKI) with activity against Raf kinase and several receptor tyrosine kinases [2]. Treatment of advanced HCC has made further progress with the introduction of regorafenib and lenvatinib [3, 4].

However, considering the mechanism of pharmacological action, these TKIs cannot respond to emergencies, such as tumor rupture. In the past, Yu et al. [5] reported the use of direct hepatic artery puncture in transarterial therapy for liver cancer under ultrasonographic guidance. They performed radioembolization, but they reported multiple punctures, and the method of the puncture technique was not clearly stated. We reported 2 cases in Japanese in the journal of the Japan Society of Hepatology where we succeeded in performing direct hepatic artery puncture [6, 7]. The Japanese Society of Hepatology has granted consent to report our puncture technique used in these cases in English papers.

\section{Case Presentation}

\section{Case 1}

Our first report was of a 69-year-old male with a clinical diagnosis of HCC (BCLC stage B). His liver function was ALBI grade 1, so we considered TACE to be the most reasonable first therapy. However, his celiac artery was occluded from a previous surgical procedure. We then attempted to approach from the mesenteric artery, which was immediately impassable due to a thrombus. Thus, we performed direct hepatic artery puncture under ultrasonographic guidance and, although temporarily, we were able to control the disease with percutaneous lipiodol chemotherapy.

Case 2

Our second case was an 81-year-old female with hepatitis C virus-related HCC (BCLC stage A). She had been transported by ambulance with emergent ruptured HCC. Her liver function was ALBI grade 1. In this case, her tumor-feeding artery diverged from the cystic artery; selective TACE would have been very difficult and she refused surgery. Therefore, we performed direct hepatic artery puncture, and after confirming insertion into the blood vessel with solenoid echo, we performed ethanol injection to destroy the feeding artery. This treatment successfully stopped tumor bleeding, and furthermore, tumor necrosis was obtained, as seen on an enhanced computed tomography image.

\section{Procedure of Direct Hepatic Artery Puncture}

We injected drugs into the artery with only one puncture. Due to the recent improvement in the resolution of ultrasound equipment, identification of thin arteries was possible by color Doppler and contrast ultrasound. It is common to use an attachment when puncturing under ultrasound guidance; however, it is difficult to handle slight deviations and puncture a single point with this method, and it is not suitable for slight changes in puncture direction. Thus, we first drew the target artery in one plane as far as possible with the abdominal ultrasound image. We then approached the target blood vessel at an approximate angle of $20^{\circ}$ on the same plane by the freehand method and stopped just before puncturing the blood vessel. 
Takashima et al.: Direct Hepatic Artery Puncture for Hepatocellular Carcinoma

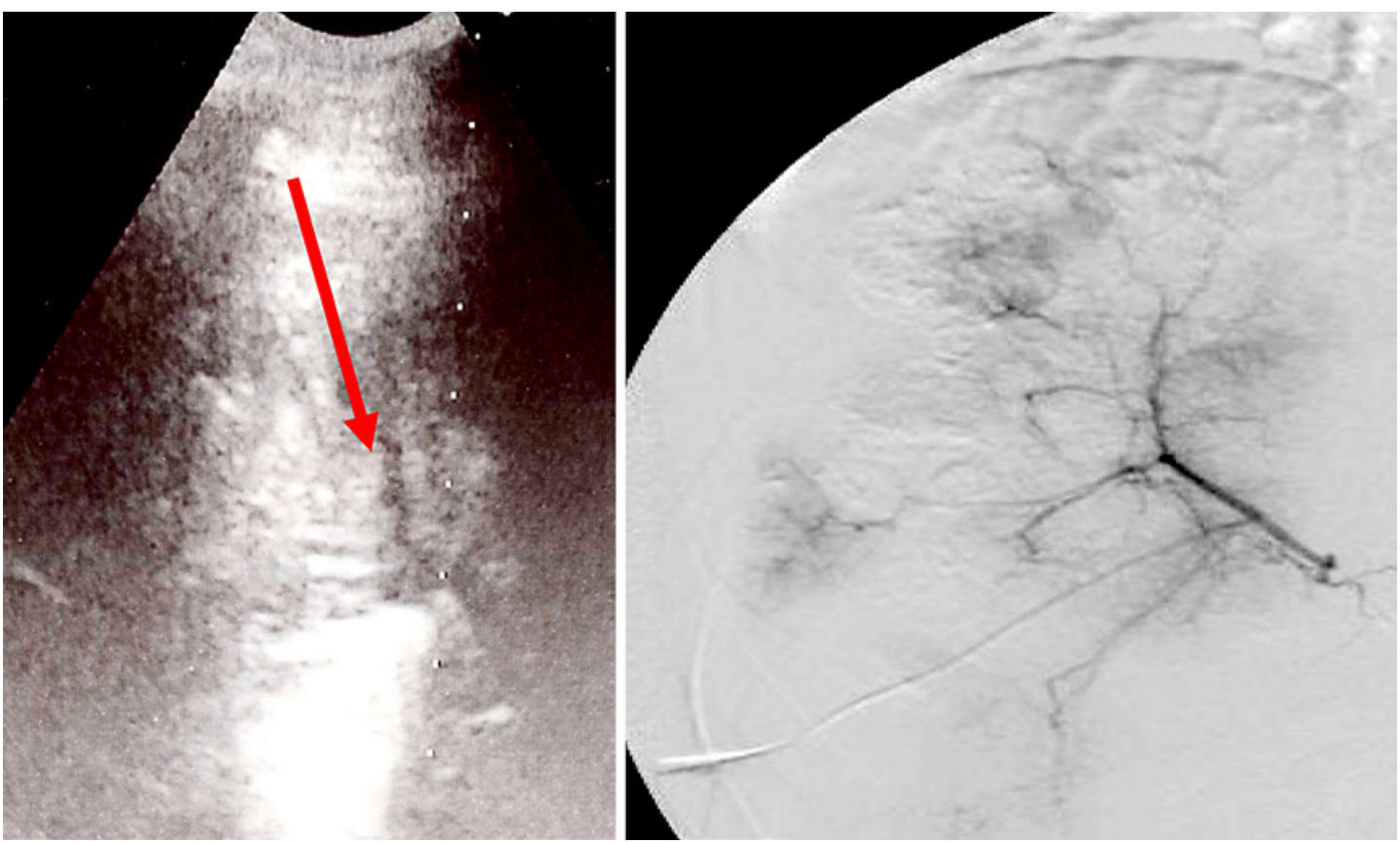

Fig. 1. Case 1. Tumor staining. The red arrow indicates the puncture route (left). Tumor staining is seen in contrast after insertion of the external needle (right).

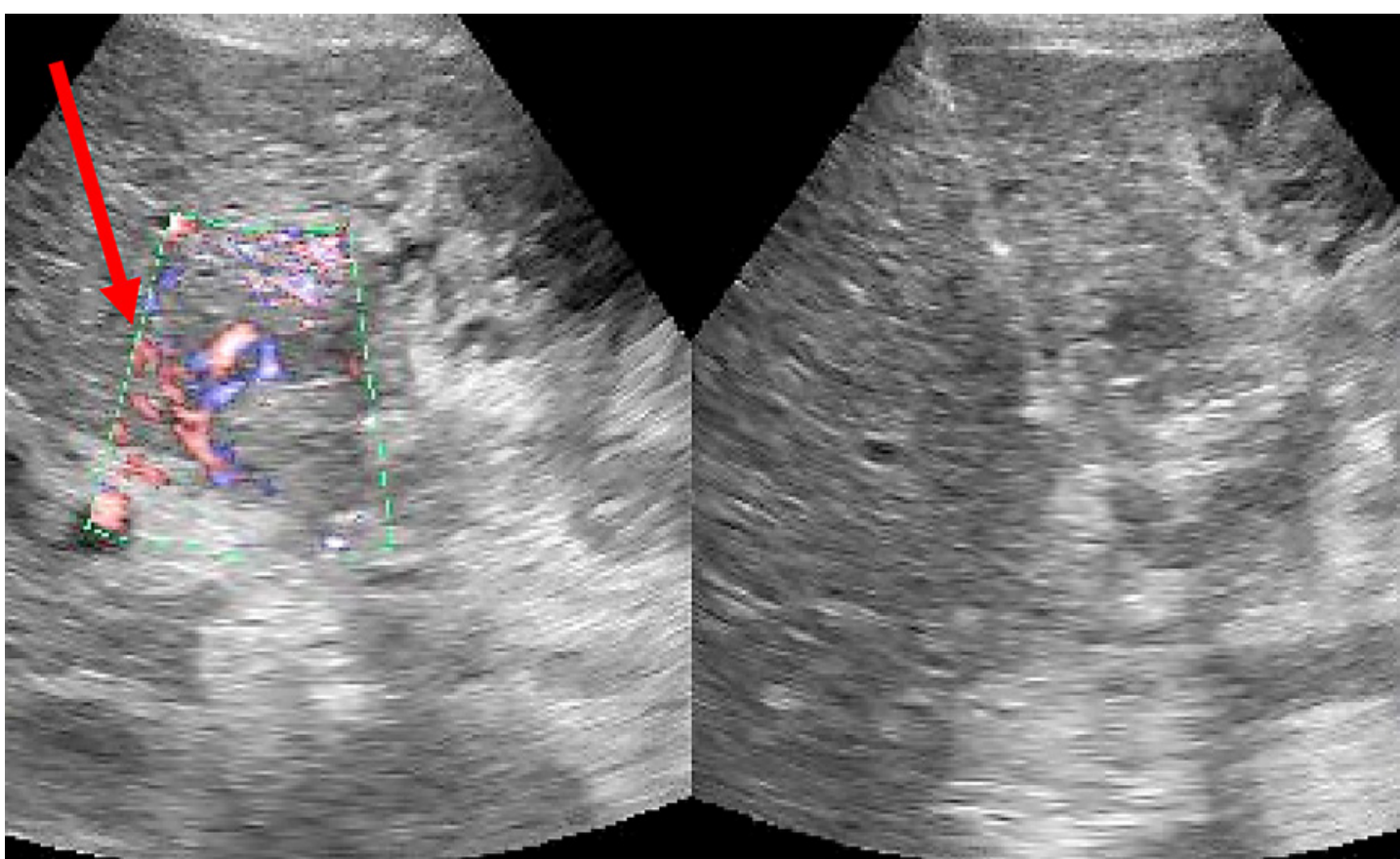

Fig. 2. Case 2. Color Doppler echo can identify the feeding artery of the tumor. We injected ethanol directly into the nutrient vessel (red arrow). 
Finally, we adjusted to the patient's breath and punctured the artery at a speed to overcome the elasticity of the blood vessels.

Case 1 underwent the procedure in the angiography room. We inserted a 21-G elastane needle into the blood vessel, which was confirmed by backflow of blood and angiography after plastic stent insertion (Fig. 1). In case 2, the purpose was to stop the hemorrhage of a ruptured HCC in an elderly patient who did not wish to undergo surgery, so the procedure was performed at the bedside without X-ray equipment (Fig. 2). We inserted a 21-G ethanol injection needle into the blood vessel, which was confirmed by backflow of blood into the needle.

\section{Discussion}

Here, we described a new, remarkable method of direct artery puncture. TACE is the standard treatment for intermediate-stage HCC. TKI therapy is indicated for HCC refractoriness to TACE, defined as an insufficient therapeutic effect after $\geq 2$ procedures [8]. However, there are cases in which such treatment is difficult anatomically. Occasionally, an emergency such as HCC rupture may occur, in which case TACE is the treatment of choice. Spontaneous rupture is a serious complication of HCC, and it is critical to determine whether hemostasis can be achieved. Even among these cases, there may be instances in which anatomical problems prevent embolization. Transarterial embolization is an alternative when no other treatment options, such as surgery, are available.

Previously, Imamura et al. [9] reported the possibility of treating hepatic infarction by injecting ethanol into the hepatic artery for the treatment of HCC. In recent years, there have been doubts about whether hepatic infarction is desirable to maintain functional hepatic reserve, but an intra-arterial ethanol injection is very effective for hemostasis when HCC ruptures. As previously mentioned, both our cases were reported in Japanese in the journal of the Japan Society of Hepatology in 2011 [6] and 2017 [7] and involved only one puncture, performed under the guidance of ultrasound. In contrast, Yu et al. [5] reported a multiplepuncture method. Hepatic artery puncture is dangerous, so it is very important to succeed with only a single puncture.

Our method requires advanced puncture techniques. Furthermore, considering complications, such as bleeding and aneurysm formation, this is not the treatment of choice if there are other, safer alternatives available. However, it can be considered as an option if there are no other viable, effective treatments.

\section{Acknowledgments}

Special thanks to Kohoku Hospital and the Osaka General Hospital of West Japan Railway Company for their support of direct hepatic puncture and equipment.

\section{Statement of Ethics}

This study was carried out in accordance with the Declaration of Helsinki. These cases were urgent and could not be reviewed by the hospital's ethics committee, but the procedures were performed with the consent of all members at the cancer board of our institution. Additionally, both patients provided written informed consent. 


\section{Disclosure Statement}

The authors have no conflicts of interest to declare.

\section{Funding Sources}

The authors received no funding from any organization.

\section{Author Contributions}

Hidetaka Takashima: formulation and execution of treatment methods; Michihisa Moriguchi, Kohichiroh Yasui, Natsuko Hayashi, Kyohei Ikeda, Kiyoshi Ogiso, Chihiro Yokomizo, Hirokazu Uejima, Hideo Tomioka, Tadashi Itoh, Shigeto Mizuno, Seiji Shimizu, and Yoshito Itoh: advice on treatment strategies.

\section{References}

1 Schicho A, Pereira PL, Michalik K, Beyer LP, Stroszczynski C, Wiggermann P. Safety and efficacy of transarterial chemoembolization with degradable starch microspheres (DSM-TACE) in the treatment of secondary liver malignancies. Onco Targets Ther. 2018 Jan;11:345-50.

2 Liu L, Cao Y, Chen C, Zhang X, McNabola A, Wilkie D, et al. Sorafenib blocks the RAF/MEK/ERK pathway, inhibits tumor angiogenesis, and induces tumor cell apoptosis in hepatocellular carcinoma model PLC/PRF/5. Cancer Res. 2006 Dec;66(24):11851-8.

3 Bruix J, Qin S, Merle P, Granito A, Huang YH, Bodoky G, et al. Regorafenib for patients with hepatocellular carcinoma who progressed on sorafenib treatment (RESORCE): a randomised, double-blind, placebocontrolled, phase 3 trial. Lancet. 2017 Jan;389(10064):56-66.

4 Kudo M, Finn RS, Qin S, Han KH, Ikeda K, Piscaglia F, et al. Lenvatinib versus sorafenib in first-line treatment of patients with unresectable hepatocellular carcinoma: a randomised phase 3 non-inferiority trial. Lancet. 2018 Mar;391(10126):1163-73.

5 Yu M, Lewandowski RJ, Ibrahim S, Riaz A, Ryu RK, Benito A, et al. Direct hepatic artery puncture for transarterial therapy in liver cancer. J Vasc Interv Radiol. 2010 Mar;21(3):394-9.

6 Kida N, Takashima H, Nishikawa T, Tomikashi K. Percutaneous transhepatic artery chemolipiodolization for multiple hepatocellular carcinomas. Acta Hepatol Japon. 2011 Jan;52(10):687-94.

7 Kikuchi K, Takashima H, Mori T, Yokomizo C. Percutaneous transhepatic intra-arterial ethanol injection therapy for ruptured hepatocellular carcinoma. Acta Hepatol Japon. 2017 Sept;58(9):510-8.

8 Furuta M, Moriguchi M, Okuda K, Kataoka S, Mizuno N, Takemura M, et al. Impact of insufficient response with an increase in tumor number in predicting transcatheter arterial chemoembolization refractoriness for hepatocellular carcinoma. Dig Dis. 2018 Jul;36(5):385-94.

9 Imamura M, Shiratori Y, Sato S, Obi S, Okudaira T, Teratani T, et al. Percutaneous hepatic infarction therapy for hepatocellular carcinoma. AJR Am J Roentgenol. 1998 Oct;171(4):1031-5. 